International Journal of Engineering, Science and Technology

Vol. 3, No. 1, 2011, pp. 298-304
INTERNATIONAL

JOURNAL OF

ENGINEERING,

SCIENCE AND

TECHNOLOGY

www.ijest-ng.com

(C) 2011 MultiCraft Limited. All rights reserved

\title{
Representation of a Lie Group $G(0,1)$ and Incomplete 2D Hermite polynomials
}

\author{
M. J. S. Shahwan
}

Department of Mathematics, University of Bahrain , P.O. Box: 32038, KINGDOM OF BAHRAIN

${ }^{*}$ Corresponding Author: e-mail: :dr_mohannad69@yahoo.com,

\section{Abstract}

In this paper we derive some generating relations involving Incomplete 2D Hermite polynomials (I2DHP) $h_{m, n}(x, y ; \tau)$, of two-variable ,two index and one parameter using Lie-theoretic approach .Certain (known or new) generating relations for the polynomials related to I2DHP are also obtained as special cases.

Keywords: Incomplete 2D Hermite polynomials , Lie algebra , generating relations.

(2010) MSC : 33C45, 33C50,33C80

\section{Introduction}

Recently, Dattoli (2003) and Dattoli et al. (1997) introduced the Incomplete 2D Hermite polynomials (I2DHP) $h_{m, n}(x, y ; \tau)$, and discussed their properties and their explicit representations and applications. Their link with Laguerre polynomials was discussed and it was shown that they are a useful tool to study quantum mechanical harmonic oscillator entangled states.

The I2DHP, $h_{m, n}(x, y ; \tau)$ are characterized by two-variable, two index and one parameter and specified by the series (Dattoli et al., 1997)

$$
h_{m, n}(x, y, \tau)=m ! n ! \sum_{r=0}^{\min (m, n)} \frac{\tau^{r} x^{m-r} y^{n-r}}{r !(m-n) !(n-r) !}
$$

are linked to the

$$
h_{m, n}(x, y)=m ! n ! \sum_{r=0}^{\min (m, n)} \frac{x^{m-r} y^{n-r}}{r !(m-n) !(n-r) !}
$$

by the relation

$$
h_{m, n}(x, y ; \tau)=\tau^{\frac{(m+n)}{2}} h_{m, n}\left(\frac{x}{\sqrt{\tau}}, \frac{y}{\sqrt{\tau}}\right)
$$

Furthermore they are linked to the Laguerre polynomials by the relation (Dattoli et al., 1997).

$$
h_{n, m}(x, y ; \tau)=n ! \tau^{n} x^{m-n} L_{n}^{m-n}\left(-\frac{x y}{\tau}\right), m>n
$$


where $L_{n}^{m-n}(x)$ denotes associated Laguerre Polynomials .

Very recently, Subuhi et al. (2008) obtained some implicit summation formulae for I2DHP by using differential analytical means on their respective generating functions .In Shahwan (2009), the author derived generating functions of I2DHP, $h_{m, n}(x, y ; \tau)$ through Weisner's (Weisner , 1955) method. The present sequel to some of these earlier papers is motivated largely by the aforementioned works of Dattoli (Dattoli, 2003; Dattoli et al.,1997). We aim here at presenting a general formula involving generating function for I2DHP which may be considered a further contribution to the theory of I2DHP. We consider the problem of framing I2DHP, $h_{m, n}(x, y ; \tau)$ into the context of the representation $\uparrow_{\omega, \mu}$ of a four-dimensional Lie algebra $\varsigma(0,1)$ (Miller, 1968). Generating relations involving $h_{m, n}(x, y ; \tau)$ and associated Laguerre Polynomials, $L_{l}^{(n)}(x)$ (Rainville, 1971) are also obtained. Thus, all of the identities obtained here will be given an explicit group-theoretic interpretation instead of being considered merely as the result of some formal manipulation of infinite series.

\section{Representation $\uparrow_{\omega, \mu}$ of $\varsigma(0,1)$ and generating relations}

We note that the following isomorphism (Miller, 1968)

$$
\varsigma(0,1) \cong L[G(0,1)]
$$

where $L[G(0,1)]$ is the Lie algebra of a complex four-dimensional Lie group $G(0,1)$, a multiplicative matrix group with elements (Miller, 1968)

$$
g(a, b, c, \tau)=\left(\begin{array}{cccc}
1 & c e^{\tau} & a & \tau \\
0 & e^{\tau} & b & 0 \\
0 & 0 & 1 & 0 \\
0 & 0 & 0 & 1
\end{array}\right), a, b, c, \tau \in C
$$

The group $G(0,1)$ is called the complex harmonic oscillator group (Miller, 1968).A basis for $L[G(0,1)]$ is provided by the matrices (Miller, 1968)

$$
\begin{aligned}
& j^{+}=\left(\begin{array}{cccc}
0 & 0 & 0 & 0 \\
0 & 0 & 1 & 0 \\
0 & 0 & 0 & 0 \\
0 & 0 & 0 & 0
\end{array}\right) \quad, \quad j^{-}=\left(\begin{array}{cccc}
0 & 1 & 0 & 0 \\
0 & 0 & 0 & 0 \\
0 & 0 & 0 & 0 \\
0 & 0 & 0 & 0
\end{array}\right) \\
& j^{3}=\left(\begin{array}{llll}
0 & 0 & 0 & 1 \\
0 & 1 & 0 & 0 \\
0 & 0 & 0 & 0 \\
0 & 0 & 0 & 0
\end{array}\right) \quad, \quad \varepsilon=\left(\begin{array}{llll}
0 & 0 & 1 & 0 \\
0 & 0 & 0 & 0 \\
0 & 0 & 0 & 0 \\
0 & 0 & 0 & 0
\end{array}\right)
\end{aligned}
$$

with commutation relations

$$
\left[j^{3}, j^{ \pm}\right]= \pm j^{ \pm},\left[j^{+}, j^{-}\right]=-\varepsilon,\left[\varepsilon, j^{ \pm}\right]=\left[\varepsilon, j^{3}\right]=\Theta
$$


The machinery constructed in (Miller , 1968)will be applied to find a realization of the irreducible representation $\uparrow_{\omega, \mu}$ of $\varsigma(0,1)$, where $\omega, \mu \in C$ such that $\mu \neq 0$. The spectrum $S$ of $\uparrow_{\omega, \mu}$ is the set $S=\{-\omega+k, k-$ anonnegativeint eger $\}$. In particular, we are looking for the function $f_{m, n}(x, y, p, s ; \tau)=Z_{m, n}(x, y ; \tau) p^{m} s^{n}$ such that

$$
\begin{aligned}
& J^{3} f_{m, n}=m f_{m, n} \quad E f_{m, n}=\mu f_{m, n} \\
& J^{+} f_{m, n}=\mu f_{m, n+1} \quad J^{-} f_{m, n}=(n+\omega) f_{m, n-1} \\
& C_{0,1} f_{m, n}=\left(J^{+} J^{-}-E J^{3}\right) f_{m, n}=\mu \omega f_{m, n}
\end{aligned}
$$

for all $n \in S$.The commutation relations satisfied by the operators $J^{ \pm}, J^{3}, E$ are

$$
\left[J^{3}, J^{ \pm}\right]= \pm J^{ \pm},\left[J^{+}, J^{-}\right]=-E,\left[J^{ \pm}, E\right]=\left[J^{3}, E\right]=0
$$

The number of possible solutions of Eq.(2.5) is tremendous. We assume that these operators take the form

$$
\begin{aligned}
& J^{+}=s\left[y+\tau \frac{\partial}{\partial x}\right] \\
& J^{-}=\frac{1}{s} \frac{\partial}{\partial y} \\
& J^{3}=s \frac{\partial}{\partial s} \\
& E=1
\end{aligned}
$$

and note that these operators satisfy the commutation relations (2.5).

We can assume $\omega=0$ and $\mu=1$ without any loss of generality for the theory of special functions. In terms of the functions $Z_{m, n}(x, y ; \tau)$ relations (2.4) become

$$
\begin{array}{cc}
\left(y+\tau \frac{\partial}{\partial x}\right) Z_{m, n}(x, y ; \tau)=Z_{m, n+1}(x, y ; \tau), & \\
\left(\frac{\partial}{\partial y}\right) Z_{m, n}(x, y ; \tau)=n Z_{m, n-1}(x, y ; \tau), & \\
\left(\tau \frac{\partial^{2}}{\partial x \partial y}+y \frac{\partial}{\partial y}-n\right) Z_{m, n}(x, y ; \tau)=0, & n=0,1,2, \ldots
\end{array}
$$

Again, if we take the function $f_{m, n}(x, y, p, s ; \tau)=Z_{m, n}(x, y ; \tau) p^{m} s^{n}$ such that

$$
\begin{aligned}
& J^{3^{\prime}} f_{m, n}=n f_{m, n} \quad E^{\prime} f_{m, n}=\mu f_{m, n} \\
& J^{+^{\prime}} f_{m, n}=\mu f_{m+1, n} \quad J^{-^{\prime}} f_{m, n}=(m+\omega) f_{m-1, n} \\
& C_{0,1}^{\prime} f_{m, n}=\left(J^{+^{\prime}} J^{-^{\prime}}-E^{\prime} J^{3^{\prime}}\right) f_{m, n}=\mu \omega f_{m, n}
\end{aligned}
$$

for all $m \in S$,then the differential operators $J^{ \pm^{\prime}}, J^{3^{\prime}}, E^{\prime}$ are given by 


$$
\begin{aligned}
J^{+^{\prime}} & =p\left[x+\tau \frac{\partial}{\partial y}\right] \\
J^{\prime^{\prime}} & =\frac{1}{p} \frac{\partial}{\partial x} \\
J^{3^{\prime}} & =p \frac{\partial}{\partial p} \\
E^{\prime} & =1
\end{aligned}
$$

and satisfy the commutation relations identical to (2.5).

Just as before taking $\omega=0$ and $\mu=1$, relation (2.8) become

$$
\begin{gathered}
\left(x+\tau \frac{\partial}{\partial y}\right) Z_{m, n}(x, y ; \tau)=Z_{m+1, n}(x, y ; \tau), \\
\left(\frac{\partial}{\partial x}\right) Z_{m, n}(x, y ; \tau)=m Z_{m-1, n}(x, y ; \tau), \\
\left(\tau \frac{\partial^{2}}{\partial y \partial x}+x \frac{\partial}{\partial x}-m\right) Z_{m, n}(x, y ; \tau)=0, \\
\end{gathered}
$$

We see from (2.7) and (2.10) that $Z_{m, n}(x, y ; \tau)=h_{m, n}(x, y ; \tau)$ where $h_{m, n}(x, y ; \tau)$ is given by (1.1).

The functions $f_{m, n}(x, y, p, s ; \tau)=h_{m, n}(x, y ; \tau) p^{m} s^{n}, n \in S$, form a basis for a realization of the representation $\uparrow_{0,1}$ of $\varsigma(0,1)$.This realization of $\varsigma(0,1)$ can be extended to a local multiplier representation $T(g), g \in G(0,1)$ defined on $F$ the space of all functions analytic in a neighborhood of the point $\left(x^{0}, y^{0}, p^{0}, s^{0} ; \tau^{0}\right)=(1,1,1,1,1)$.

Using operators (2.6) , the local multiplier representation (Miller , 1968) takes the form

$[T(\exp a \varepsilon) f](x, y, p, s ; \tau)=\exp (a) f(x, y, p, s ; \tau)$

$\left[T\left(\exp b j^{+}\right) f\right](x, y, p, s ; \tau)=\exp (b y s) f(x+b \tau s, y, p, s ; \tau)$

$\left[T\left(\exp c j^{-}\right) f\right](x, y, p, s ; \tau)=f\left(x, y+\frac{c}{s}, p, s ; \tau\right)$

$\left[T\left(\exp \lambda j^{3}\right) f\right](x, y, p, s ; \tau)=f\left(x, y, p, s e^{\lambda} ; \tau\right)$

for $f \in F$.If $g \in G(0,1)$ has parameters $(a, b, c, \lambda)$, then

$T(g)=T(\exp a \varepsilon) T\left(\exp b j^{+}\right) T\left(\exp c j^{-}\right) T\left(\exp \lambda j^{3}\right)$

and therefore we obtain

$[T(g) f](x, y, p, s ; \tau)=\exp (a+b y s) \cdot f\left(x+b \tau s, y+\frac{c}{s}, p, s e^{\lambda} ; \tau\right)$

The matrix elements of $T(g)$ with respect to the analytic basis $f_{m, n}(x, y, p, s ; \tau)=h_{m, n}(x, y ; \tau) p^{m} s^{n}$ are the functions $A_{l k}(g)$ uniquely determined by $\uparrow_{\omega, \mu}$ of $\varsigma(0,1)$ and we obtain relations 
$\left[T(g) f_{k, n}\right](x, y, p, s ; \tau)=\sum_{l=0}^{\infty} A_{l k}(g) f_{l, n}(x, y, p, s ; \tau), k=0,1,2, \ldots$,

which simplify to the identity

$$
\exp (a+\lambda n+b y s) h_{k, n}\left(x+b \tau s, y+\frac{c}{s} ; \tau\right)=\sum_{l=0}^{\infty} A_{l k}(g) h_{l, n}(x, y ; \tau) p^{l-k}, k=0,1,2, \ldots,
$$

which may be regarded as a generating function for the matrix elements. To find an explicit expression for this element it is sufficient to compute the coefficients of p on the left hand side of (2.13).and thus the matrix element $A_{l k}(g)$ are given by (Miller , 1968)

$$
A_{l k}(g)=\exp (a+k \lambda) c^{k-l} L_{l}^{(k-l)}(-b c), k, l \geq 0 .
$$

Substituting (2.14) into (2.13), we obtain the generating relation

$$
\begin{gathered}
\exp (\text { bys }+\lambda(n-k)) h_{k, n}\left(x+b \tau s, y+\frac{c}{s} ; \tau\right)=\sum_{l=0}^{\infty} c^{k-l} L_{l}^{(k-l)}(-b c) h_{l, n}(x, y ; \tau) p^{l-k}, \\
b, c, p \in C, n, k=0,1,2, \ldots
\end{gathered}
$$

Again taking the operators (2.9) and proceeding exactly as before, we obtain the generating relation

$$
\begin{gathered}
\exp \left(b^{\prime} x p+\lambda(m-r)\right) h_{m, r}\left(x+\frac{c^{\prime}}{p}, y+b^{\prime} \tau p ; \tau\right)=\sum_{i=0}^{\infty}\left(c^{\prime}\right)^{r-i} L_{i}^{(r-i)}\left(-b^{\prime} c^{\prime}\right) h_{m, i}(x, y ; \tau) s^{i-r}, \\
b^{\prime}, c^{\prime}, s \in C, \quad m, r=0,1,2, \ldots
\end{gathered}
$$

\section{Applications}

We consider some special cases of the generating relations obtained in previous section, which yield many new and known relations for the polynomials related to L2DP.

I. Making use of (1.4) in (2.15) we get

$$
\begin{aligned}
& \exp (\text { bys }+\lambda(n-k))\left(1+\frac{b \tau \mathrm{s}}{x}\right)^{(n-k)} L_{k}^{(n-k)}\left(-\frac{x\left(1+\frac{b \tau \mathrm{s}}{x}\right) y\left(1+\frac{c}{s y}\right)}{\tau}\right) k != \\
& \sum_{l=0}^{\infty} c^{k-l} L_{l}^{(k-l)}(-b c) L_{l}^{(n-l)}\left(-\frac{x y}{\tau}\right)(\tau)^{l-n}(x)^{k-l} p^{l-k} l !, b, c, p \in C, n, k=0,1,2, \ldots
\end{aligned}
$$

Now in particular, taking $\lambda=0, \tau=1, y=-1, u=x$ and replacing $s$ and $p$ by $t$ in (3.1) we get a result of Miller (Miller , 1968)

II. Again making use of (1.4) in (2.16) we get 


$$
\begin{aligned}
& \exp \left(b^{\prime} x p+\lambda(m-r)\right)\left(x+\frac{c^{\prime}}{p}\right)^{(r-m)} L_{m}^{(r-m)}\left(-\frac{\left(x+\frac{c^{\prime}}{p}\right)(y+b \tau p)}{\tau}\right)= \\
& \sum_{i=0}^{\infty}\left(c^{\prime}\right)^{r-i} L_{i}^{(r-i)}\left(-b^{\prime} c^{\prime}\right)(x)^{i-m} L_{m}^{(i-m)}\left(-\frac{x y}{\tau}\right) s^{i-r}, b^{\prime}, c^{\prime}, s^{\prime} \in C, m, r=0,1,2, \ldots
\end{aligned}
$$

Again in particular taking $p=s=\tau=1, \lambda=0$ a and replacing $x$ by $-c_{1}, y$ by $b_{1}, b^{\prime}$ by $b_{2}, c^{\prime}$ by $-c_{2}, m$ by $l$, $r$ by $l+n$ and $i$ by $j$ in (3.2) we get a result of (Miller, 1968).

It may be remarked that matrix elements computed in (2.14) are entire analytic functions of the group parameters .it will be more convenient to derive more results of Hermite and Laguerre polynomials by choosing special values of the parameters and variable.

\section{Conclusions}

We have considered the problem of framing I2DHP,$h_{m, n}(x, y ; \tau)$ into the context of the representation $\uparrow_{\omega, \mu}$ of the Lie algebra $\varsigma(0,1)$ of the complex harmonic group $G(0,1)$. Generating relations involving I2DHP are obtained by using Miller's technique. Some relations for the products of Laguerre polynomials and identities of Miller are also obtained as special cases.

Further, we observe that these operators $J^{-}, J^{+} J^{-^{\prime}}, J^{+^{\prime}}$ and $I=1$ satisfy the following commutation relations,

$$
\begin{aligned}
& {\left[J^{-}, J^{+}\right]=\left[J^{{{ }^{\prime}}^{\prime}}, J^{+^{\prime}}\right]=I,} \\
& {\left[J^{-}, J^{+^{\prime}}\right]=\left[J^{-^{\prime}}, J^{+}\right]=0,} \\
& {\left[J^{-}, J^{-^{\prime}}\right]=\left[J^{+}, J^{+^{\prime}}\right]=0 .}
\end{aligned}
$$

These relations imply that the five operators $J^{-}, J^{+}{J^{-}}^{\prime}, J^{+^{\prime}}, I$ are closed with regard to the commutation relations. Therefore ,they form a realization of an abstract five-dimensional Lie algebra which is the Lie algebra of the Heisenberg-Weyl group $W(2, R)$ or to its complex extension $W(2, C)$ for a two-mode system. Thus I2DHP form a certain basis for this realization of the Heisenberg-Weyl algebra $\omega(2, R)$ or to its complex extension $\omega(2, C)$ see (Wunsche , 1998) and the references therein .By the quadratic combinations of the basic operators $J^{-}, J^{+} J^{-^{\prime}}, J^{+^{\prime}}, I$, we can form ten more operators , which form several Lie Algebras.

The study of the I2DHP for applications as well as for its connections with various Lie algebras is an interesting problem for further research. Recently, the interest in the generalized Hermite polynomials and their popularity have increased considerably in view of their important role and applications in Bose-like operator calculus. See, for example, Rosenblum (1994). We will take up derivation of recurrence relations and generating functions for these polynomials in our forthcoming paper.

\section{Acknowledgement}

The author would like to gratefully acknowledge the financial support of Deanship of Scientific research , University of Bahrain.

\section{References}

Dattoli G.,2003 . Incomplete 2D Hermite polynomials: properties and applications,J.Math.Anal.Appl.Vol.284 , pp.447-454.

Dattoli G., Lorenzutta S. , Maino G. and Torre A.,1997. Theory of multi variable Bessel functions and Hermite polynomials , Matematiche LII , pp.179-197.

Rosenblum M.,1994. Generalized Hermite polynomials and the Bose-like oscillator calculus, Oper. Theory Adv.Appl., 73, Birkhauser,Basel.

Miller W.Jr.,1968. Lie theory and special functions , Academic press , New York and London .

Miller W.Jr.,1972. Symmetry Groups and their Applications, Academic Press, New York, London..

Rainville E.D.,1971. Special Functions ,Macmillan Co. INCNew York ,1960;Reprinted by Chelsea Publ. Co., Bronx, New York. 
Shahwan, M.J.S., 2009. Weisner's method to obtain generating functions for the Incomplete 2D Hermite polynomials, J.Assoc.Arab Univ.Bas.Sci.Vol.7, pp.115-122.

Subuhi Khan , Pathan,M.A. ,Nader Ali Makboul Hassan and Ghazala Yasmin, 2008. Implicit summation formulae for Hermite and related polynomial , J.Math.Anal.Appl., Vol.344, pp.408-416.

Weisner L., 1955. Group-theoretic origin of certain generating functions, Pacific J. Math.,Vol. 5, pp.1033-1039.

Wunsche A., 1998. Laguerre 2D-functions and their applications in quantum optics , J.Phys.A.:Math.Gen.Vol.31,pp.8267-8287.

Biographical notes

M.J.S.Shahwan received his Ph.D. degree in Mathematics from Rajasthan University ,Jaipur, India in 1996 . He is currently an Associate Professor at College of Science, Department of Mathematics, University of Bahrain. His research interest is in Lie theory and Special functions. He has more than 15 research publications

Received June 2011

Accepted June 2011

Final acceptance in revised form June 2011 